\title{
Numerical simulation of EHD flows using Discontinuous Galerkin Finite Element methods
}

\author{
P.A. Vázquez ${ }^{\mathrm{a}, *}$, A. Castellanos ${ }^{\mathrm{b}}$ \\ a Dpto. de Fúsica Aplicada III, E.S.I. \\ Camino de los Descubrimientos s/n, 41092 Sevilla, Spain \\ ${ }^{b}$ Dpto. de Electrónica y Electromagnetismo, Facultad de Física \\ Avda. Reina Mercedes s/n, 41012 Sevilla, Spain
}

\begin{abstract}
The aim of this paper is to explore the capability of Discontinuous Galerkin Finite Element methods to solve numerically the charge transport equation in EHD convective flows, in both strong and weak injection regimes. These methods are especially suited to treat purely hyperbolic problems, as it is the charge transport equation in most EHD problems. We consider the 2D electroconvective flow between two parallel plates. We compare our computations with the analytical results in the hydrostatic regime, the linear and non-linear stability analysis, computing both the electric and velocity fields. The stability of the finite amplitude electroconvection is also analysed. Comparisons are made with computations in the literature obtained with other numerical techniques. The results show that DG-FEM are a very good alternative to simulate numerically EHD convective flows.
\end{abstract}

Keywords: Electrohydrodynamics, Discontinuous Galerkin, Stability

\footnotetext{
*Corresponding author

Email address: pvazquez@us.es (P.A. Vázquez)
} 


\section{Introduction}

Electrohydrodynamics (EHD) deals with the interaction of fluids, electric charges and electric fields[1]. It is an interdisciplinary area with important applications in industrial processes such as the manufacture of electrostatic precipitators, refrigeration of high voltage transformers, etc[2]. Specifically, EHD involving fluids is important in the development of pumping devices in microelectromechanical systems (MEMS), bio MEMS and nanotechnology applications $[3,4,5,6]$.

In this paper we explore the application of Discontinuous Galerkin Finite Element methods to the numerical simulation of EHD problems. In particular, we analyze numerically the classical EHD problem of the 2D flow between two parallel plates immersed in a dielectric liquid for both strong and weak injection regimes. When a high voltage is applied between the parallel plates, the electrodes inject electric charge into the liquid, and the Coulomb force put the liquid into motion. Experiments and theoretical analysis show that the pattern of convection in $3 \mathrm{D}$ is made of hexagonal cells similar to those of Rayleigh-Bénard convection, while in 2D the structure of the flow is made of two-dimensional rolls $[7,8]$. The onset of the global motion is controlled by a non dimensional parameter involving the applied electric potential, the mobility of the charge carriers and the properties of the fluid.

Several numerical methods have been used to analyse this problem. The fist attempt was made by Castellanos, Atten and Perez[9]. They used first finite difference based methods to solve all the equations, but they found that the strong numerical diffusion introduced invalidates the method due to the hyperbolic nature of the charge transport equation. To solve this 
issue, they turned to Particle-In-Cell methods(PIC)[10, 11] to deal with the charge transport equation. In this method the electric charge is modelled as 'superparticles', that moves upon the action of the electric and velocity fields computed on a mesh. PIC have been extensively used in plasma physics, and are known to introduce very little numerical diffusion. This technique has been applied since then to EHD convection problems by the authors both in $2 \mathrm{D}[12,13]$ and $3 \mathrm{D}[14]$. It has been proved to be able to describe accurately the electric charge distribution in EHD convective problems. However, it has its drawbacks. It is very expensive computationally, specially in 3D geometries. Also the injection mechanism needed to maintain a given value of charge density at the injector is quite delicate.

Others method have been applied to deal with the hyperbolic nature of the charge transport equation. In two papers $[15,16]$ the authors, in collaboration with Georghiou, have compared the PIC technique with a FCT method with corrections to minimize the numerical diffusion. Both techniques give similar results for the linear stability criterion and the amplitude of the velocity roll, although the distribution of electric charge differs. Also, there are oscillations that appear with PIC (absent with FCT).

In two recent papers[17, 18] Traoré et Pérez have used a finite volume approach combined with a total vaiation diminishing scheme (TVD) to solve the equation transport problem. In the reference [18] they consider the EHD electroconvection between two parallel plates. They analyze the linear and non-linear stability criteria, the finite amplitude electroconvection and the dependence of the electric Nusselt number on the parameters of the problem. All these computations are made for the strong injection regime. They obtain 
very good results, reproducing the expected structure of the electric charge density and a qualitative agreement with the experimental behaviour of the electric Nusselt number.

A small drawback of finite volume methods is the construction of the mesh for complicated geometries. In that regard, finite element methods are known for its ability to adapt easily to complicated geometries. In this paper, we explore the application of Finite Elements Discontinuous Galerkin methods to solve numerically the charge transport equation[19]. These methods were originally developed to deal with hyperbolic problems, although in recent years have been applied to all kind of problems involving partial differential equations[20].

The paper is organized as follows. In section 2 we state the problem and the relevant non-dimensional parameters. In section 3 we describe the numerical procedures used for each magnitude. Then in section 4 we discuss the results of our computations. We compare the computed values of the linear stability threshold with the values obtained theoretically $[7,8]$ for both the strong and weak injection regimes. Then we consider the finite amplitude convection. First we analyze the computed distribution of electric charge, in order to verify the capability of the numerical method to reproduce the steep gradients of electric charge. We study the hysteresis problem in the finite amplitude regime, computing the non-linear stability critical value of the stability parameter for the strong injection case. Finally, we consider the stability of the finite amplitude regime for values of the stability parameter higher than the linear threshold. The conclusions are presented in section 5. We will see that DG methods are able to reproduce closely the analytical 
results and the computations made with other numerical methods.

\section{Problem formulation}

We consider two plane electrodes a distance $d$ apart immersed in a nonconductive fluid. An electric potential is applied between the plates so that injection of charge occurs. The electric field forces the charges away from the injector and in this way a space charge appears. The Coulomb force pushes the charges, and the liquid with them. If the electric potential is high enough all the liquid is put into motion. Here we analyze the 2D case, so the system is considered to be infinite along one of the direction parallel to the electrodes.

The fluid is incompressible, isothermal and insulating with mass density $\rho$, kinematic viscosity $\nu$ and permittivity $\varepsilon$. Due the small values of the electric current, the heating is negligible and these magnitudes can be taken as constants. An electric voltage $\Phi_{0}$ is applied between the plates. The charge carriers are considered to be of an unique type, with ionic mobility $K$. Unipolar autonomous injection is assumed, that is, injection of charge occurs only at the bottom plate, the density of charge at the injector is constant and equal to $q_{0}$, and the ions discharge instantaneously once they reach the opposite electrode.

There are three mechanisms responsible for the motion of ions: drift by the electric field $\left(\mathbf{J}_{E}=K q \mathbf{E}, K\right.$ being the ionic mobility, $q$ the electric charge density and $\mathbf{E}$ the electric field), convection by the fluid $\left(\mathbf{J}_{c}=q \mathbf{u}, \mathbf{u}\right.$ being the velocity of the fluid), and molecular diffusion $\left(\mathbf{J}_{d}=-D \nabla q, D\right.$ being the diffusivity). In EHD, diffusion is only important inside a very thin boundary 
layer near the electrodes, and it is not relevant for phenomena developing in the bulk [1]. So in our case the electric charge is transported only by the electric and velocity fields. The problem becomes purely hyperbolic, implying the existence of sharp gradients of electric charge density. Therefore the current density is given by $\mathbf{J}=q(K \mathbf{E}+\mathbf{u})$.

The scales for all the involved variables are

$$
\begin{array}{lll}
x, y \sim d & \Phi \sim \Phi_{0} & E \sim \Phi_{0} / d \\
u \sim K \Phi_{0} / d & t \sim d^{2} / K \Phi_{0} & p \sim \rho K^{2} \Phi_{0}^{2} / d^{2} \\
q \sim \varepsilon \Phi_{0} / d^{2} & &
\end{array}
$$

$p$ being the pressure.

The non-dimensional equations defining the problem are

$$
\begin{gathered}
\nabla^{2} \Phi=-q, \quad \mathbf{E}=-\nabla \Phi \\
\nabla \cdot[q(\mathbf{u}+\mathbf{E})]+\frac{\partial q}{\partial t}=0, \\
\nabla \cdot \mathbf{u}=0 \\
\frac{\partial \mathbf{u}}{\partial t}+(\mathbf{u} \cdot \nabla) \mathbf{u}=-\nabla p+\frac{M^{2}}{T} \nabla^{2} \mathbf{u}+M^{2} q \mathbf{E} .
\end{gathered}
$$

The non-dimensional parameters of the problem are

$$
T=\frac{\varepsilon \Phi_{0}}{\rho \nu K}, \quad C=\frac{q_{0} d^{2}}{\varepsilon \Phi_{0}}, \quad M=\frac{1}{K} \sqrt{\frac{\varepsilon}{\rho}} .
$$

$T$ is the ratio of the force term to the viscous term, and will be the stability parameter. $M$ is the ratio of the hydrodynamic mobility to the ionic mobility[21] and $C$ measures the injection strength.

In the linear stability analysis, the threshold value for the onset of the motion depends on the wavelength of the perturbation[7]. The minimum of 
these values is the absolute linear stability threshold. The minimum wavelength depends on the injection parameter $C$. In the case of strong injection $(C=10)$, the critical wavelength turns out to be $k_{\min }=5.113$. For weak injection $(C=0.1)$ it is $k_{\min }=4.573$. We consider as domain a rectangle of size $L=\pi / k_{\min }, k_{\min }$ being the value corresponding to each case. This way, we solve the problem in one half of a convective cell. The non-dimensional domain and boundary conditions are shown in figure 1. At the lateral walls the perpendicular components of the electric and velocity field are null. The value of the charge density at the injector (the bottom plate) is $C$.

\section{Numerical algorithms}

We use Discontinuous Galerkin Finite Elements (DG-FEM) to solve the charge transport equation. These methods were originally developed to deal with hyperbolic problems, although in recent years have been applied to all kind of problems involving partial differential equations[19, 20]. The key idea is to consider internal degrees of freedom inside every element. This means that the computed magnitudes inside each element do not have to be continuous across the elements borders. The connection between elements is achieved using the so called numerical fluxes. In this way, conservation is imposed locally. These methods have proved to be very stable when treating hyperbolic problems, and allow to work with complex geometries, as well as prescribing different orders of approximation inside each element.

We use a third order polynomial approximation inside each DG element. The order of the polynomial approximation has a great influence in the outcome of the computations, as will be shown in the paper. Our numerical 
experiments show that the third order is a good compromise between accuracy and computational cost.

For the choice of the numerical flux, let us point out that, although the global problem is non-linear, the charge transport equation is linear at each iteration, as the electric and velocity fields are computed at each time step. In this linear case all the usual numerical fluxes available in the literature (Godunov, Engquit-Osher, Lax-Friedrichs) become equivalent to the so called full upwind flux. Therefore, at each face the value of the numerical flux of a magnitude is given by the flux of the element where the total ionic velocity comes from. The total ionic velocity is the sum of the electric and velocity fields $\mathbf{v}_{\text {ion }}=\mathbf{u}+\mathbf{E}$.

We are applying a very simplified scheme with no slope limiter. So, although the full upwind scheme gives good results, small oscillations still may appear in the regions of strong gradients of electric charge. In this way, non-physical negative values of the electric charge may occur due to these oscillations. In order to correct these non-physical values, at each time step, if negative values of the charge density occur we change them to zero. The time scheme is a backward Euler in order to enhance stability. The resulting numerical scheme is first order in time.

Both the electric field and the velocity field are computed using Continuous Galerkin Finite Elements(CG-FEM). We solve the Navier-Stokes equation using a Incremental Pressure Correction Scheme (IPCS)[22]. We consider second order elements for the electric potential(CG-FEM) and the velocity field (CG-FEM). The pressure is approximated using first order CGFEM, in order to comply with the LBB condition. The IPCS scheme is first 
order in time.

In all the simulations the time-step is chosen so that the Courant number is always lower than one, changing it during the computations if necessary. The Courant number is defined as

$$
\text { Cou }=\frac{v_{\max } \Delta t}{h_{\min }} .
$$

Here, $v_{\max }$ is the maximum value of the total ionic velocity and $h_{\min }$ is the size of the smallest element.

The algorithms have been implemented using the DOLFIN[23] Python library. This is an interface to FEniCS[24], a framework for automated solution of differential equations by the Finite Element method. The library is open sourced under the LGPL license and is freely available from the FEniCS website.

\section{Results and discussion}

\subsection{Hydrostatic regime}

In order to verify the ability of the DG-FEM method to simulate the charge distribution, and to choose the best mesh to deal with each injection case, we have run simulations in the hydrostatic regime, that is, without computing the velocity of the fluid. At the beginning of the computation the domain has no electric charge. As time progresses, a front of charge advances from the bottom electrode towards the top electrode, pushed by the electric field. After some units of non-dimensional time, a steady state is attained, with distributions of electric charge density and electric field that admit an analytical solution. We compare the computed steady distributions of charge and the electric field with these analytical solution. 
In the weak injection regime $(C=0.1)$ the lateral length of the domain is $L=0.687$. The mesh is made of triangles built upon a uniform grid with 40 intervals along each direction. The total number of triangular elements is 3200, with 1681 vertices. Figure 2 shows the charge density along a vertical line at non-dimensional time $t=0.4$. The steady analytical solution is also plotted. We can see that the DG-FEM is able to describe this front of charge with no spurious oscillation near the region of the steepest gradient, although some numerical diffusion is introduced by the upwind scheme. Figure 3 shows the computed and analytical charge densities along a vertical line when the steady state has been reached. The maximum difference between the computed value of the charge density and the analytical solution is $0.4 \times$ $10^{-5 \%}$. Also the values of the electric current computed at the injector and the collector differ in $1.4 \times 10^{-5 \%}$.

In the strong regime case $(C=10)$ it is $L=0.614$. The mesh is uniform with 40 intervals along the $\mathrm{X}$ direction, while for the $\mathrm{Y}$ direction it is thinner near the bottom and coarser near the top electrode. It has 4000 elements and 2091 vertices. Figure 4 shows the computed and analytical charge densities for $C=10$ along a vertical line when the steady state has been reached. The maximum difference between the computed value of the charge density and the analytical solution is $0.12 \%$. Also the values of the electric current computed at the injector and the collector differ in $0.10 \%$.

\subsection{Linear stability criteria}

The theoretical linear stability analysis studies the stability of the flow against small perturbations. The magnitudes of the problem are considered to grow exponentially with a growth factor depending on the stability param- 
eter $T$. When this growth factor is negative the system is stable, and when it is positive a convective roll develops. The critical value $T_{c}$ corresponds to a zero growth factor. The theory shows that the critical value $T_{c}$ does not depend on the mobility parameter $M$.

We have run a set of simulations changing the value of the stability parameter $T$ for different fixed values of the mobility parameter $M$, for both strong $(C=10)$ and weak $(C=0.1)$ injection regimes. As initial condition for the charge density, we set the analytical profile for the hydrostatic regime. Then we compute the electric field and solve the Navier-Stokes equation. The electric and velocity fields obtained are used to advance the charge density. The process is repeated iteratively in time.

\subsubsection{Linear stability criterion in the strong injection regime}

Figure 5 plots the evolution in time for $M=20$ and several values of $T$ of the global angular momentum of the convective cell, computed as $A M=\int\left|\left(\mathbf{r}-\mathbf{r}_{0}\right) \times \mathbf{u}\right| d \mathrm{~S}$, where $\mathbf{r}_{0}$ points to the center of the domain. This magnitude gives an idea of the strength of the velocity roll. For all values of $T$ the growth becomes exponential in a certain interval of time (this corresponds to the linear sections of the curve in the figure, as the scale of the $Y$ axis is logarithmic). In this region the angular momentum is considered to depend on time as $A M=A e^{\sigma t}$, where $\sigma$ is the growth factor. Using a linear fit for the function $\sigma(T)$ the value of $T_{c}$ corresponding to $\sigma=0$ is obtained for the different values of $M$. Table 1 shows the values of $T_{c}$ from our simulations for four different values of $M$. These numbers are to be compared with $T_{c}^{a}=164.1$, the critical value obtained from the linear stability analysis, independent of the value of $M[7]$. The error of our computed 
Table 1: Critical values for the onset of instability from the simulations for several values of $M$. The value obtained from the linear stability analysis is $T_{c}^{a}=164.1$, independent of $M$.

\begin{tabular}{rl}
$M$ & $T_{c}$ \\
\hline 5 & 163 \\
10 & 163 \\
20 & 163 \\
100 & 163
\end{tabular}

values, estimated from the fitting parameters, is of the order of $8 \%$, so the theoretical value is inside the error margin for all values of $M$. The average of the computed values differs from the theoretical value in $0.67 \%$. Also, the criterion is independent of $M$, within the error margins, as it is predicted by the theoretical analysis.

\subsubsection{Linear stability criterion in the weak injection regime}

We have run a similar set of simulations for the weak injection regime $(C=0.1)$. The numerical procedure is exactly the same as in the strong injection regime. The only difference is the mesh, that it is the same used in the hydrostatic computation for $C=0.1$. However, a comment must be made about the choice of the mesh. In this regime, the theoretical value of $T_{c}$ is 24148 . So, for a mobility parameter $M=20$ the Reynolds number at the onset of the instability is expected to be $R e=T / M^{2}=60$, and this uniform mesh is able to capture the velocity field. On the other hand, for $M=5$ the expected value of the Reynolds number when the rolls starts to develop is $R e=966$, and the mesh is too coarse. As a matter of fact we observe 
that the computations diverge for these small values of $M$. However, this mesh still allows to compute the linear stability threshold for small values of $M$, because the exponential growth of the magnitudes is very slow, and can be observed even for very low values of the maximum velocity, before the computation diverges.

Figure 6 plots the evolution in time of the total angular momentum for $T=25000$ and $M=20$. The convective roll takes much more time to develop that for the strong injection regime. This is expected, as the typical time for the roll to appear scales with $T / M^{2}[12]$. Once the roll develops, it becomes unstable. We discuss this later in the paper. Let us stress that this behaviour have been observed in [16], where both Particle-In-Cell and FCT methods have been used for computing the charge transport equation.

Figure 7 plots, using a log scale for the $Y$ axis, the evolution in time of the total angular momentum for $M=20$ and different values of $T$. The exponential growth during the early stages of the simulation is clearly observed. As in the strong injection case, we obtain the growth factor $\sigma(T)$ from the slopes of these curves. Using a linear fit, we get the value of $T_{c}$ corresponding to a growth factor $\sigma=0$. Table 2 shows the values of $T_{c}$ obtained for four values of $M$. These numbers are to be compared with $T_{c}^{a}=24148$, the critical value obtained from the linear stability analysis, independent of the value of $M[7]$. The average of the computed values differs from the theoretical value in $0.38 \%$. Also, the maximum relative difference among the computed values is $0.50 \%$, which is consistent with the independence with $M$ of the linear stability criterion. The error of our computed values of $T_{c}$, estimated from the fitting parameters, is of the order of $2 \%$, so the theoretical value is inside 
Table 2: Critical values for the onset of instability from the simulations for several values of $M$ in the weak injection regime. The value obtained from the linear stability analysis is $T_{c}^{a}=24148$, independent of $M$.

\begin{tabular}{rl}
$M$ & $T_{c}$ \\
\hline 5 & 24000 \\
10 & 24050 \\
20 & 24046 \\
100 & 24121
\end{tabular}

the error margins.

\subsection{Finite amplitude electroconvection}

The precedent sections show the DG methods are able to produce a very accurate estimation of the critical value of the linear stability analysis for both strong and weak injection. However, the ability of the method to deal with the non-linear features of the problem has to be tested too. In this section we present three results related to the non-linear features of EHD electroconvection.

\subsubsection{Distribution of electric charge}

If the value of $T$ is greater than the critical value $T_{c}$ a velocity roll appears. Figure 8 show the contour plot of the stream function for $M=20$ and $t=15$. The velocity roll is fully developed here, with a maximum velocity greater than the electric field $\left(u_{\max }=3.33\right.$ for $T=170$ and $M=10$, while the electric field is $E_{\max } \simeq 1$ ). Then, in the bulk, the charge distribution is controlled mainly by the velocity field. This results in a central region empty of electric charge. This is a very characteristic feature of electroconvective 
flows, and it is important for the numerical method to be able to reproduce this structure. Figure 9 shows the distribution of electric charge density for $M=20$ and $t=15$. The central region empty of charge is clearly seen.

In figures 10 and 11 we plot the electric charge density along the central vertical line and the central horizontal line, respectively. For comparison purposes, along with the results of the computations for this paper (in red color online), we plot the charge distribution for the same lines obtained with PIC and FCT from reference [15]. In that paper, the velocity field was computed with an imposed velocity roll, so slightly different values for the charge density are obtained. But it is interesting to compare the ability of the different methods to describe the structure of the charge density. We see that all three methods are able to reproduce the gradient of electric charge. However, in figure 11 a small nonzero value of electric charge can be seen near the left corner, for both FCT and DG computations (the results of DG computations are plotted mirrored with respect to the $\mathrm{Y}$ axis to make them agree with the imposed sense of rotation of the velocity roll in [15]) Although the value of charge density is very small, it is a clear difference with respect to PIC computations. We have observed that, for DG computations, this small value depends on the order of approximation of the DG element, when using the same mesh. This is illustrated in figure 12. Here we plot the charge density along a horizontal line for three computations with the same parameters but three different orders of polynomial approximation for the DG element. The amount of charge in the empty region decreases as the order of approximation increases. The description of the gradient of electric charge can be improved either increasing the order of polynomial 
approximation or adding elements to the mesh in the regions where sharp gradients appears. An adaptive meshing process could help to improve the computations. Anyway, as we shall see in the next section, the computations with a polynomial of order 3 give very good results when compared with the theoretical predictions.

\subsubsection{Non-linear stability for the strong injection regime}

The theoretical non-linear stability analysis shows that, when the roll is fully developped with a value of $T>T_{c}$, if now the value of $T$ is decreased, the roll persists even for values lower than $T_{c}$. In experiments, this would be equivalent to apply a value of the applied voltage above the threshold for the instability and then start decreasing the applied voltage. An hysteresis phenomenon is predicted, with a non-linear criterion for the stability parameter $T_{f}<T_{c}$. For the strong injection regime the theory predicts a value $T_{f}^{a}=110$.

We have simulated this experiment taking as initial condition the steady state obtained when $T>T_{c}$. Then we have decreased slowly the value of $T$, and waited for the system to get a new steady state. While the value of the maximum velocity in this new stationary state is greater than one (the order of magnitude of the electric field) the velocity roll is still present, but when it falls below one the roll disappears. We have plotted in figure 13 the values of the maximum velocities obtained in this process for $C=10, M=20$, taking as initial configuration the steady state corresponding to $T=170$. The computed value of the linear stability threshold is $T_{c}=163$. The roll is still stable when $T<T_{c}$ and $T>T_{f}$, as predicted in the non-linear analysis. We get a value for the non-linear criterion of $T_{f}=108.7$. In reference [18], 
Traoré and Pérez obtain a value $T_{f}=107.5$. Both of them are in excellent agreement with the theoretically predicted value of 110 .

\subsubsection{Stability of the convective roll}

In 4.2.2 we have seen that a new instability is found when the value of $T$ increases. The roll pattern becomes unstable in the weak regime for all the values of $T$ that we have computed. This fact has been described before in [16], with both PIC and FCT computations. In that paper a similar phenomena was observed in the strong injection regime. Also, in the recent paper by Traoré and Pérez[18] this instability has been discussed in detail for the strong injection case.

Figure 14 plots the evolution in time of the total angular momentum for $C=10, M=10$ and $T=300,400,500,600$. The roll is stable for $T=300$, but for $T=400$ and higher, after a time, the roll destabilizes and the angular momentum oscillates around a null value, showing the emergence of a two roll pattern. Figures 15 and 16 plot the stream function and the charge density distribution when the two roll pattern is established.

Similar results were obtained by the authors in [16]. The results obtained by Traoré and Pérez in [18] are quite similar too, the only difference being that they find that the roll is unstable for $T=300$, while in our computations it is not. This may be due to the different meshes and order of approximation used in both computations. We have run three simulations for $C=10$, $M=10$ and $T=300$, with a uniform mesh of 50x50 (the same used in [18]), for three different orders of polynomial approximation for the DG elements. Figure 17 shows the evolution in time of the maximum velocity for the different polynomial orders. We see that the behaviour is quite different. The 
roll splits in two rolls for the lower order (the split can be seen in the change of the maximum velocity). For the highest order the roll is stable, although it oscillates. These oscillations start earlier for order 2, and are bigger in magnitude. It is interesting to point out that the curve for the lower order is almost identical to the figure 17 in [18].

As for the weak injection regime, in all our simulations the roll destabilizes for all the values of $T$ above $T_{c}$ that we have tested, and for all values of $M$. This is again consistent with the computations made with PIC and FCT in [16]. However, the behaviour of the system is not the same than for the strong injection case. While for $C=10$ the two roll pattern is essentially stable, with small oscillations, in the weak injection regime the roll changes the sense of rotation. In figure 6 we can observe that the angular momentum oscillates between positive and negative values of similar absolute value. When one roll is established, a small counter-roll appears and progressively takes the place of the other one, until a new counter-roll emerges.

\section{Conclusions}

We have explored the use of Discontinuous Galerkin Finite Element methods (DG-FEM) to solve numerically the charge transport equation in the $2 \mathrm{D}$ EHD convection between parallel plates in both strong and weak injection regimes. These methods are specially suited to deal with hyperbolic problems, as it is this case due to the negligible charge diffusion. We have used a very simple implementation of DG-FEM, without slope limiter. For the computations of the electric and velocity fields we have used Continuous Galerkin Finite Element methods. 
In the hydrostatic regime, the DG-FEM method is able to describe the advancing front of charge without spurious oscillations, although introducing a certain amount of numerical diffusion. But it is able to reproduces with a very good precision the analytical solution in the steady state for both injecting regimes.

We have computed the linear stability criteria for strong and weak injection, solving the electric and velocity fields. The critical values obtained from the computations agree extremely well with the analytical solution obtained from the linear stability analysis in both cases. It turns out to be essentially independent of the value of the mobility parameter, as it is predicted by the theory.

Then we have studied the non-linear aspects of the problem. When the velocity roll develops, the computations reproduce the expected distribution of electric charge, with a bulk essentially empty, as the charge distribution is controlled by the velocity field. A small deviation is observed from the PIC computations, as a small quantity of charge enters this empty region. This effect can be reduced increasing the order of the polynomial approximation of DG elements, or refining the mesh in the regions where steep gradients are present.

As for the non-linear stability and the hysteresis predicted theoretically, our computations are able to reproduce these predictions. We obtain a value of the non-linear criterion very close to the prediction of the theoretical analysis.

We have also studied the stability of the convective roll for values of $T$ greater than the linear threshold $T_{c}$. We have reproduced the results obtained 
with PIC methods and the computations described in Traoré and Pérez. We have observed that the order of polynomial approximation in DG elements can be critical to describe accurately the behaviour of the system in this fully convective regime.

These results validate the capability of DG-FEM to simulate EHD flows, even if some numerical diffusion is present. PIC methods are the less diffusive, but they are numerically expensive and difficult to implement, especially the value of the charge density at the injector. In this regard, the availability of an open source package as FEniCS makes very easy to implement the DG-FEM method. Anyway some improvements can be envisaged in our implementation of DG-FEM. Slope limiters can be introduced, as well as higher order time integration schemes. Another possible improvement could be to apply adaptive meshing techniques to get a better description of the distribution of electric charge an reduce the computational cost. In particular, we must compute magnitudes that can be compared with experiments, as it is the electric Nusselt numbers for higher values of $T$. Also, the application of this technique to the $3 \mathrm{D}$ dimensional problem is envisioned in future works.

\section{Acknowledgements}

This work has been supported by the Spanish Ministerio de Educación y Ciencia under contract FIS2011-25161 and the Junta de Andalucía under contract P09-FQM-4584.

[1] Castellanos A, editor. Electrohydrodynamics. Springer-Verlag; 1998. ISBN 3-211-83137-1. 
[2] Cowley JM. Fundamentals of Applied Electrostatics. Wiley; 1986.

[3] Darabi J, Ohadi M, DeVoe DL. An electrohydrodynamic polarization micropump for electronic cooling applications. Journal of Microelectromechanical Systems 2001;10:98-106.

[4] Wong PK, Wang TH, Deval JH, Ho MC. Electrokinetics in micro devices for biotechnology applications. EEE/ASME Trans Mechatron 2004;9:366-76.

[5] Pearson M, Seyed-Yagoobi J. Advances in electrohydrodynamic conduction pumping. IEEE Transactions on Dielectrics and Electrical Insulation 2009;16(2):424-34.

[6] Castellanos A, Ramos A, González A, Green NG, Morgan H. Electrohydrodynamics and dielectrophoresis in microsystems: scaling laws. Journal of Physics D: Applied Physics 2003;36(20).2584

[7] Atten P, Moreau R. Stabilité électrohydrodynamique des liquides isolants soumis à une injection unipolaire. Journal de Mécanique 1972;11:471 - 520 .

[8] Atten P, Lacroix JC. Electrohydrodynamic stability of liquids subjected to unipolar injection: non linear phenomena. Journal of Electrostatics $1978 ; 5: 439-52$.

[9] Castellanos A, Atten P, Pérez AT. Finite amplitude electroconvection in liquids in the case of weak unipolar injections. PCH, PhysicoChemical Hydrodynamics 1987;9(3/4):443 -52. 
[10] Brackbill JU, Kothe DB, Ruppel HM. Flip: A low-dissipation, particlein-cell method for fluid flow. Computer Physics Communications $1988 ; 48(1): 25-38$.

[11] Hockney RW, Eastwood JW. Computer Simulations using Particles. McGraw-Hill; 1981.

[12] Chicón R, Castellanos A, Martín E. Numerical modelling of coulombdriven convection in insulating liquids. Journal of Fluid Mechanics 1997;344:43 - 66 .

[13] Vázquez PA, Soria C, Castellanos A. Numerical simulation of twodimensional ehd plumes mixing finite element and particle methods. In: Electrical Insulation and Dielectric Phenomena, 2004. CEIDP '04. 2004 Annual Report Conference on. 2004, p. $122-5$.

[14] Vázquez PA, Castellanos A. Stability analysis of the 3d electroconvective charged flow between parallel plates using the particle-in-cell method. In: Dielectric Liquids (ICDL), 2011 IEEE International Conference on. 2011, p. $1-4$.

[15] Vázquez PA, Georghiou GE, Castellanos A. Characterization of injection instabilities in electrohydrodynamics by numerical modelling: comparison of particle in cell and flux corrected transport methods for electroconvection between two plates. Journal of Physics D: Applied Physics $2006 ; 39: 2754-63$.

[16] Vázquez PA, Georghiou GE, Castellanos A. Numerical analysis of the 
stability of the electrohydrodynamic (ehd) electroconvection between two plates. Journal of Physics D: Applied Physics 2008;41(17):175303.

[17] Traoré P, Pérez AT, Koulova D, Romat H. Numerical modelling of finite-amplitude electro-thermo-convection in a dielectric liquid layer subjected to both unipolar injection and temperature gradient. Journal of Fluid Mechanics 658;:279-93.

[18] Traoré P, Pérez AT. Two-dimensional numerical analysis of electroconvection in dielectric liquid subjected to strong unipolar injection. Physics of Fluids 2012;24:037102-1 - 22 .

[19] Hesthaven JS, Warburton T. Nodal Discontinuous Galerkin Methods. Springer; 2008.

[20] Cockburn B, Shu C. Runge-kutta discontinuous galerkin methods for convection-dominated problems. Journal of Scientific Computing 2001;16(3):173-261.

[21] Felici N. Phénomenes hydro et aérodynamiques dans la conduction des diélectriques fluides. Revue General d'Electrostatique 1969;78:717 -34.

[22] Goda K. A multistep technique with implicit difference schemes for calculating two- or three-dimensional cavity flows. Journal of Computational Physics 1979;30(1):76 - 95 .

[23] Logg A, Wells GN. Dolfin: Automated finite element computing. ACM Trans Math Softw 2010;37(2):20:1-20:28. 
[24] Logg A, Mardal KA, Wells GN. Automated Solution of Differential Equations by the Finite Element Method. Springer; 2010. 


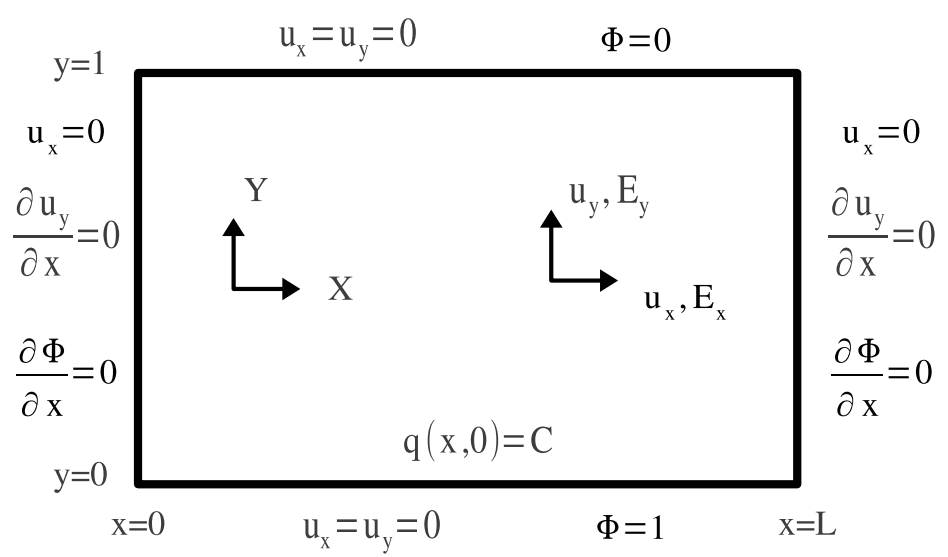

Figure 1: Non-dimensional computational domain and boundary conditions for the problem. 


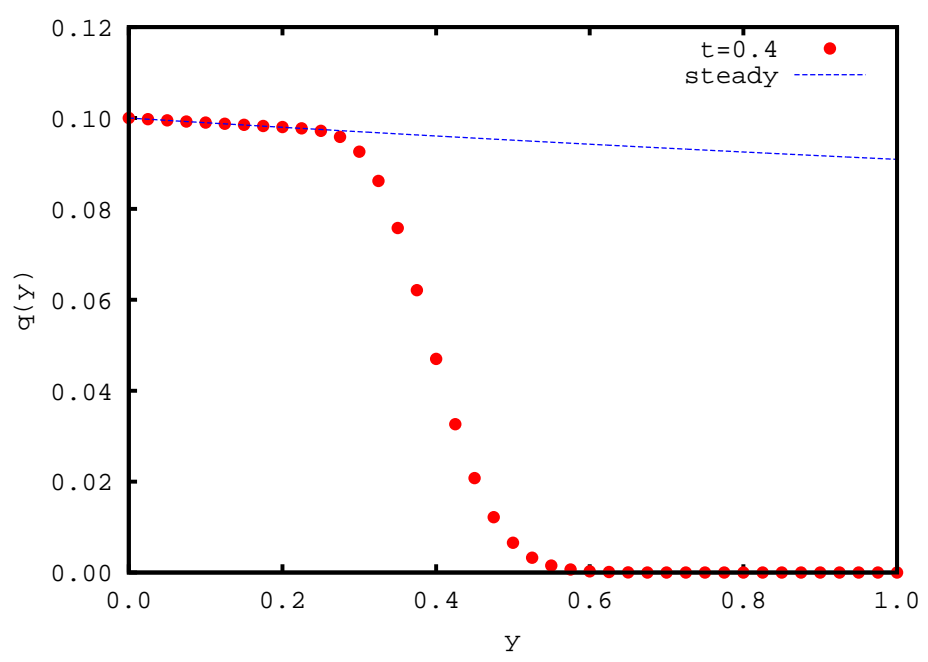

Figure 2: Charge density along the vertical central line of the domain for the hydrostatic solution for $C=0.1$ and $t=0.4$. The analytical solution for the steady state is also plotted.

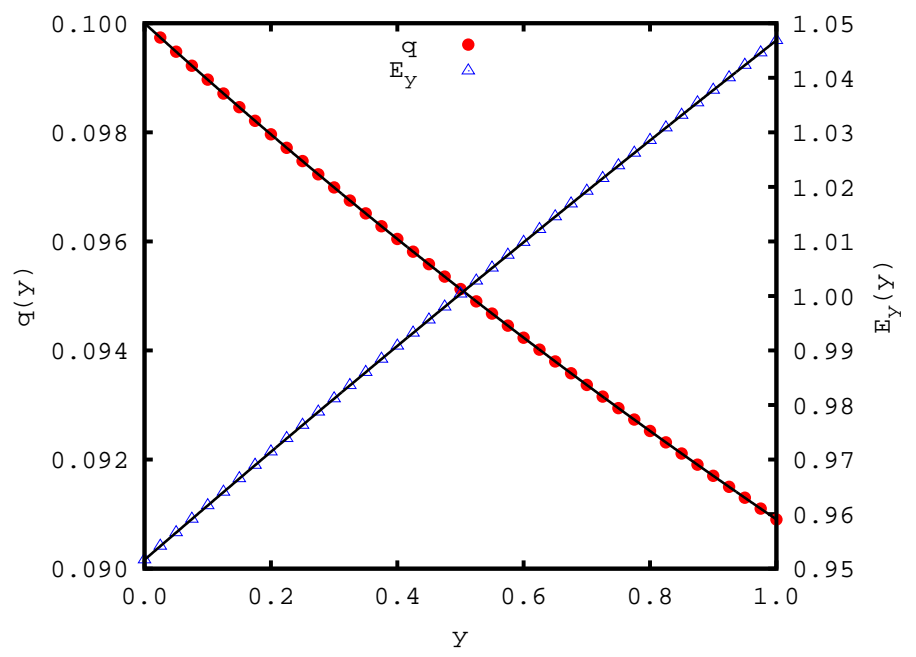

Figure 3: Charge density and vertical electric field along the vertical central line of the domain for the hydrostatic solution when the steady state is attained for $C=0.1$. The analytical solutions are also plotted. 


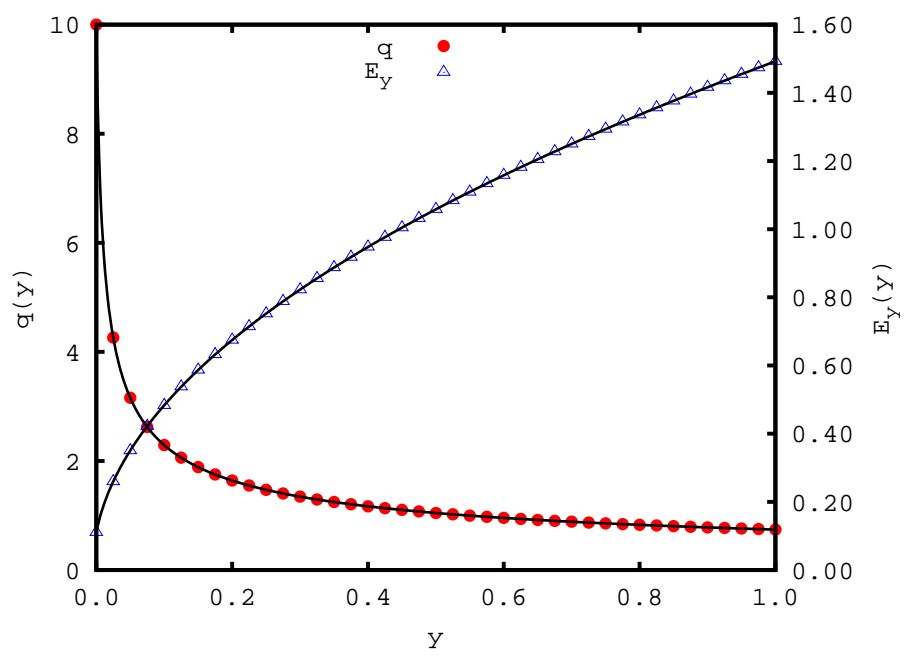

Figure 4: Charge density and vertical electric field along the vertical central line of the domain for the hydrostatic solution when the steady state is attained for $C=10$. The analytical solutions are also plotted.

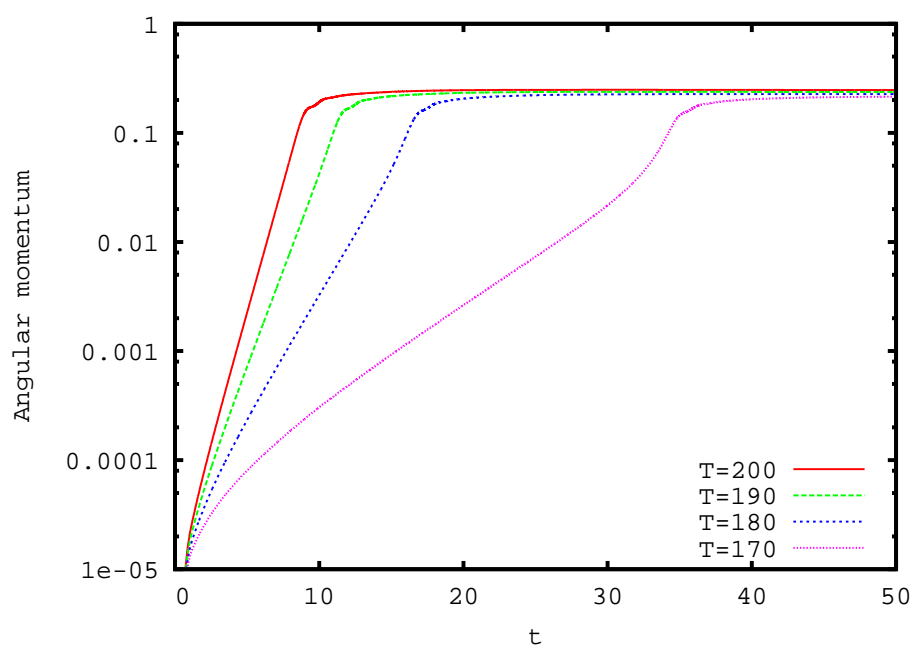

Figure 5: (color online) Evolution in time of the total angular momentum for $C=10$ and $M=20$. The critical value of $T$ can be estimated from the regions where linear growth is observed (the scale is logarithmic for the $\mathrm{Y}$ axis) 


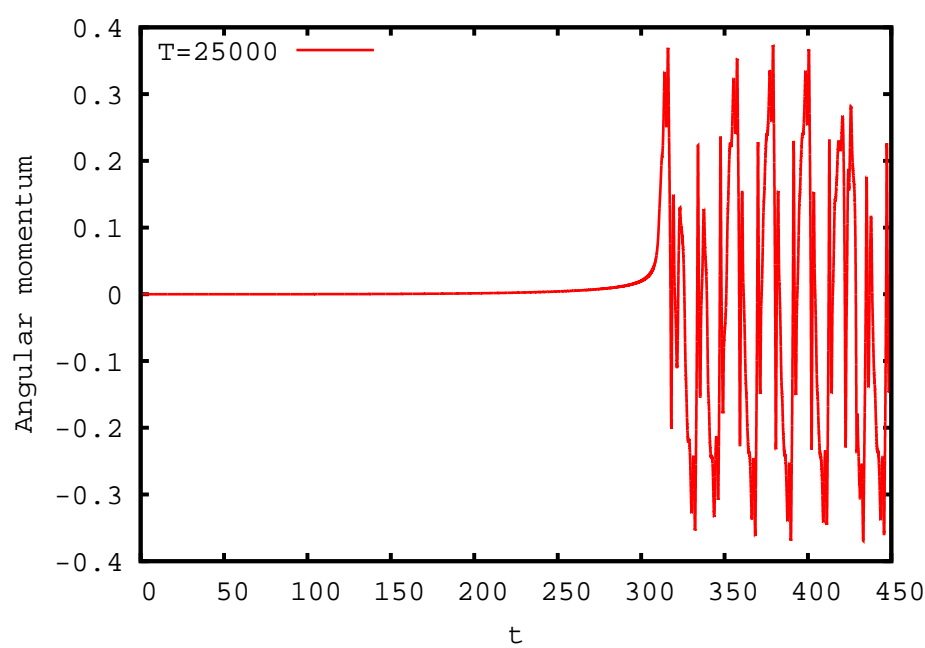

Figure 6: Evolution in time of the total angular momentum for $C=0.1, T=25000$ and $M=20$. After the velocity roll develops it becomes unstable.

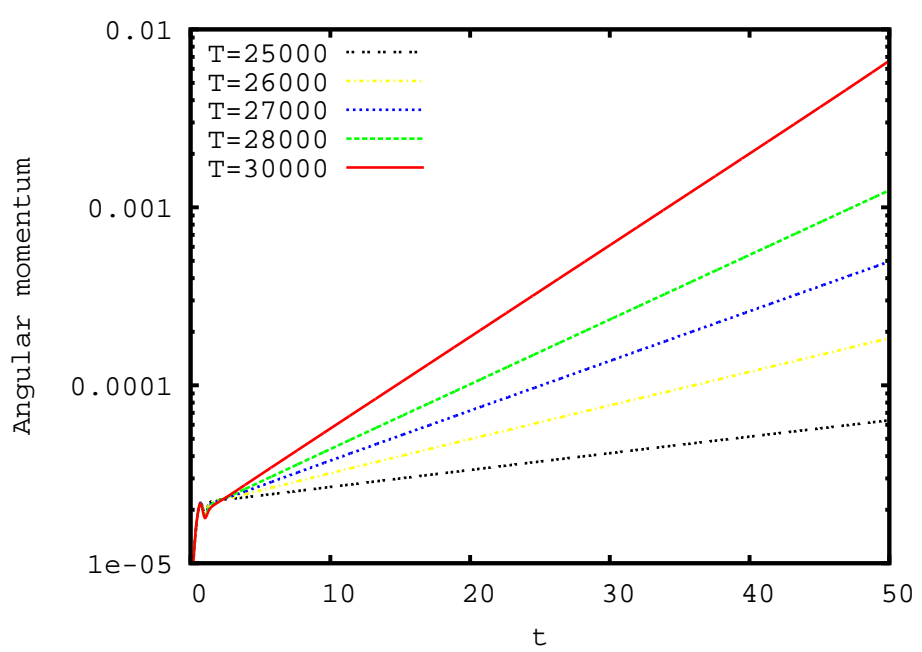

Figure 7: (color online) Exponential growth in time of the total angular momentum for $C=0.1$ and $M=20$. The critical value of $T$ can be estimated from the slopes of the curves (the scale is logarithmic for the $\mathrm{Y}$ axis) 


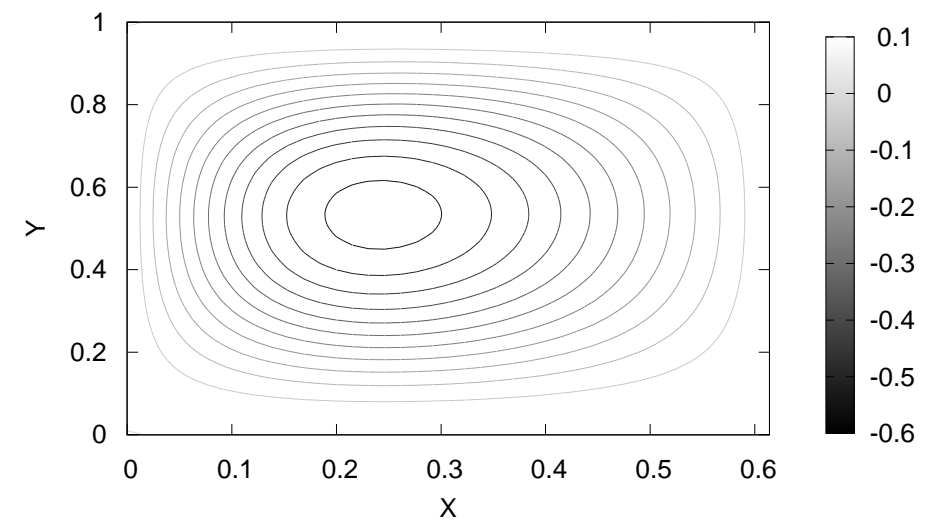

Figure 8: Contour plot of the stream function for $t=70, C=10, M=20, T=200$. The velocity roll is completely developped.

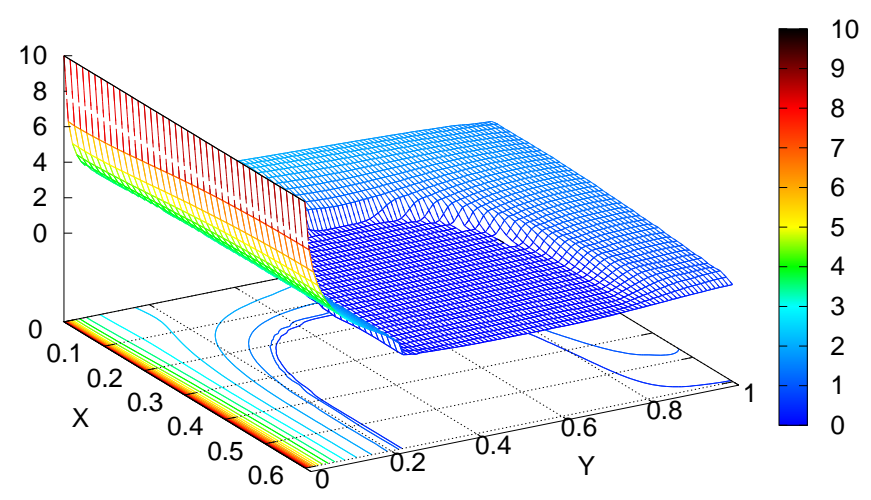

Figure 9: (color online) Charge density for $t=70, C=10, M=20, T=200$. The contour plot is plotted on the lower plane. The central region of the convective is void of electric charge. 


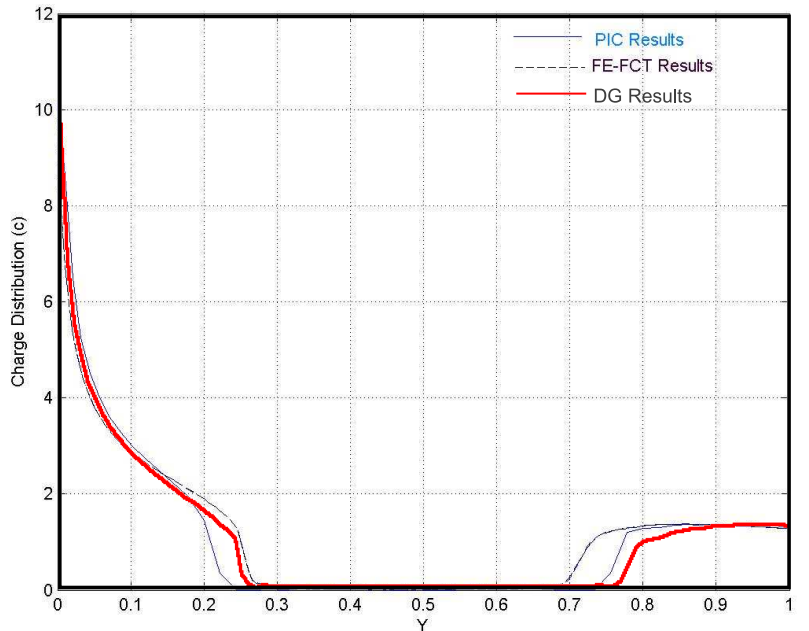

Figure 10: (color online) Electric charge density along a vertical line for $C=10, T=200$, $M=20$ and $t=70$. Results computed with PIC and FCT with an imposed velocity roll are also plotted. 


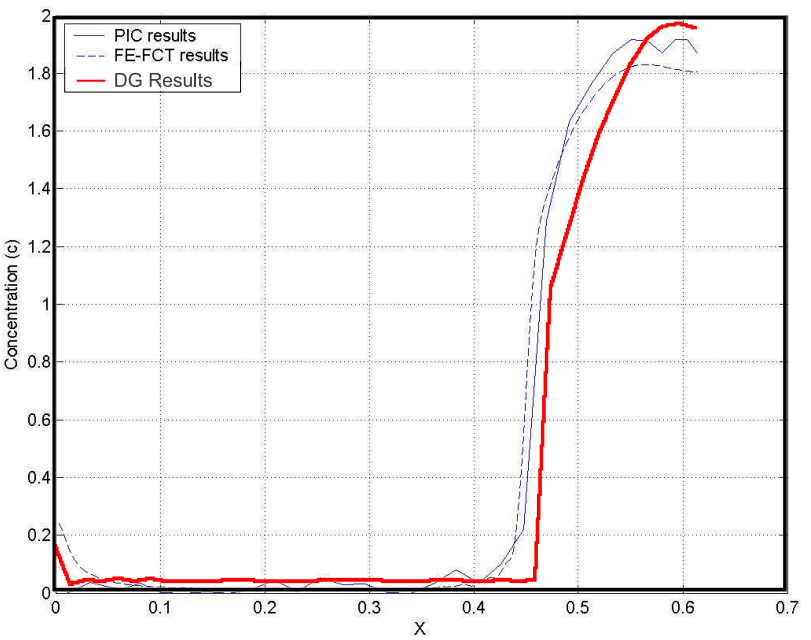

Figure 11: (color online) Electric charge density along a horizontal line for $C=10$, $T=200, M=20$ and $t=70$. Results computed with PIC and FCT with an imposed velocity roll are also plotted. A small nonzero value of charge density is observed near the left corner. 


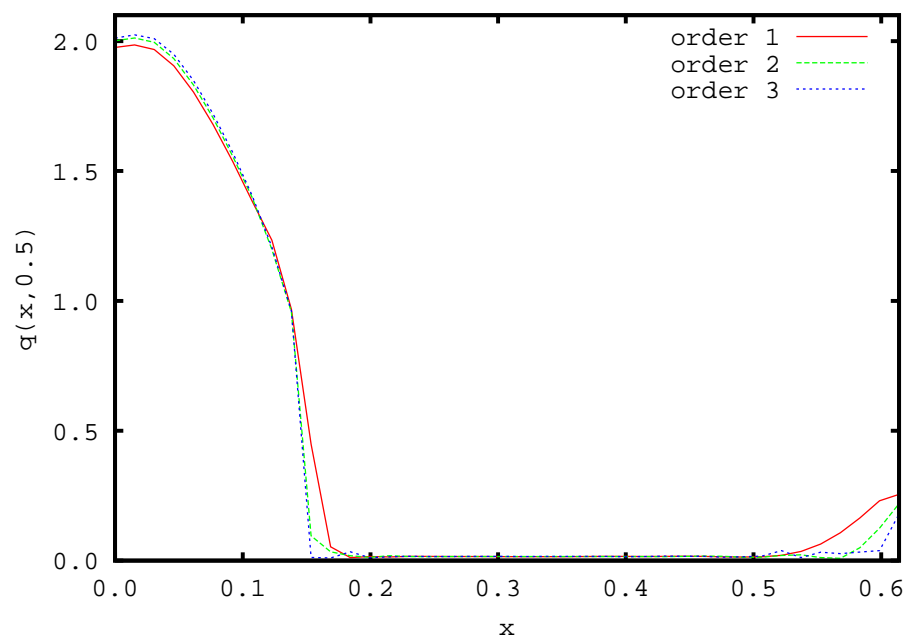

Figure 12: (color online) Electric charge density along a horizontal line for $C=10$, $T=200, M=20$ and $t=70$ for three different orders of polynomial approximation in DG-FEM. The computations have been made on the same mesh.

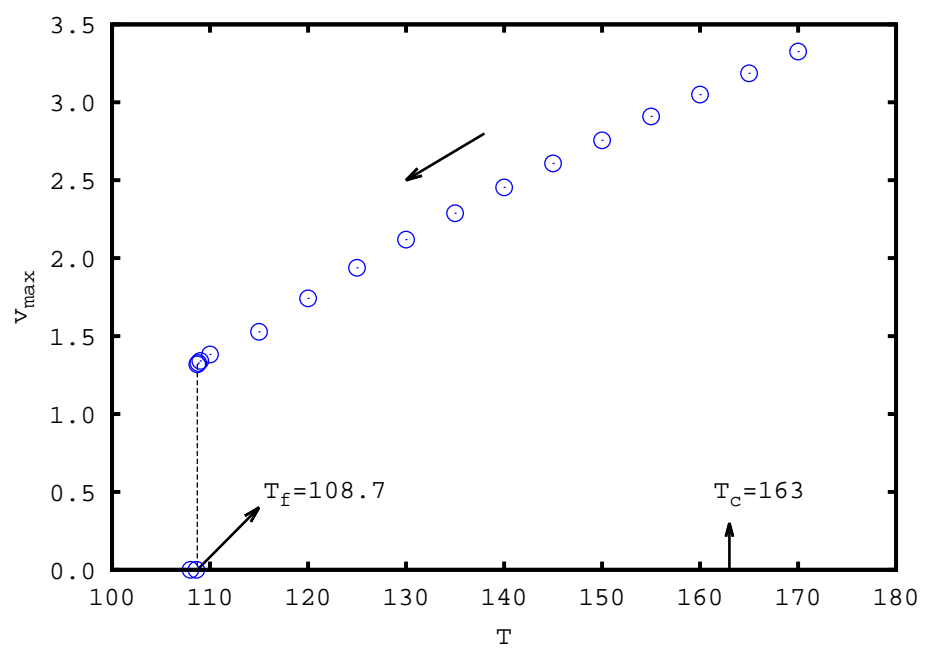

Figure 13: Hysteresis loop for $C=10, M=20$. Starting from a developped roll, when decreasing the value of $T$ the roll is still stable for $T_{f}<T<T_{c}$. The computed non-linear stability threshold is $T_{f}=108.7$. The analytical value is $T_{f}^{a}=110$. 


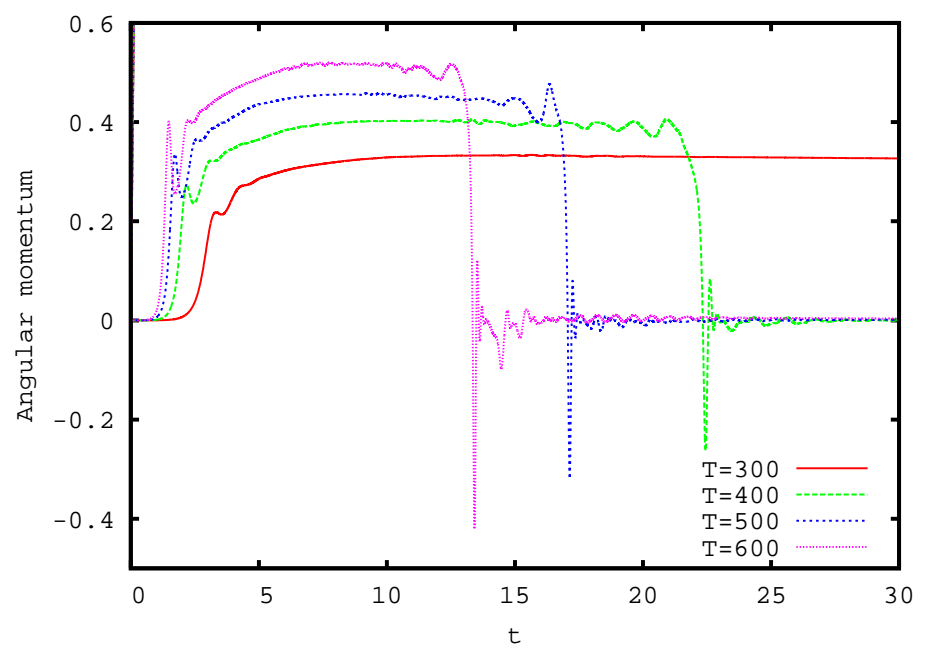

Figure 14: (color online) Evolution in time of the total angular momentum for $C=10$, $M=20$ and several values of $T$. The roll structure becomes unstable for high values of $T$.

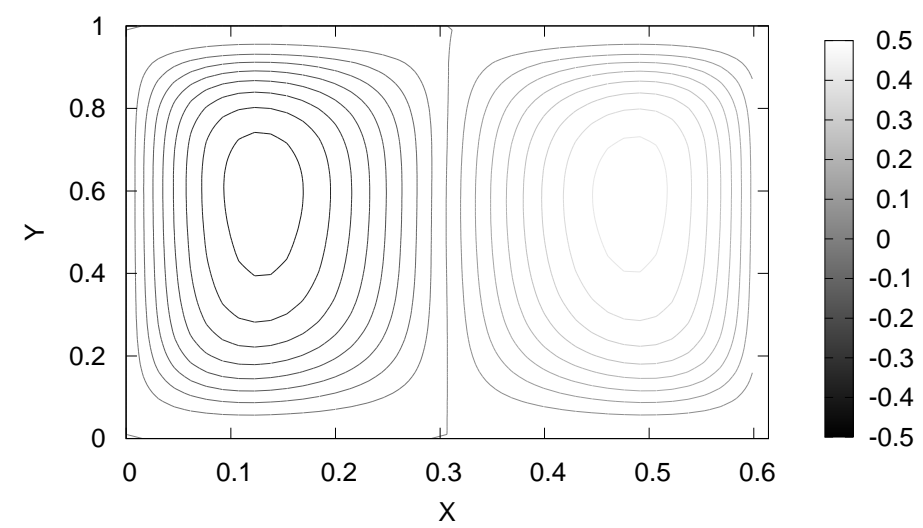

Figure 15: Contour plot of the stream function for $C=10, M=20, T=600$ and $t=20$, with the two roll structure. 


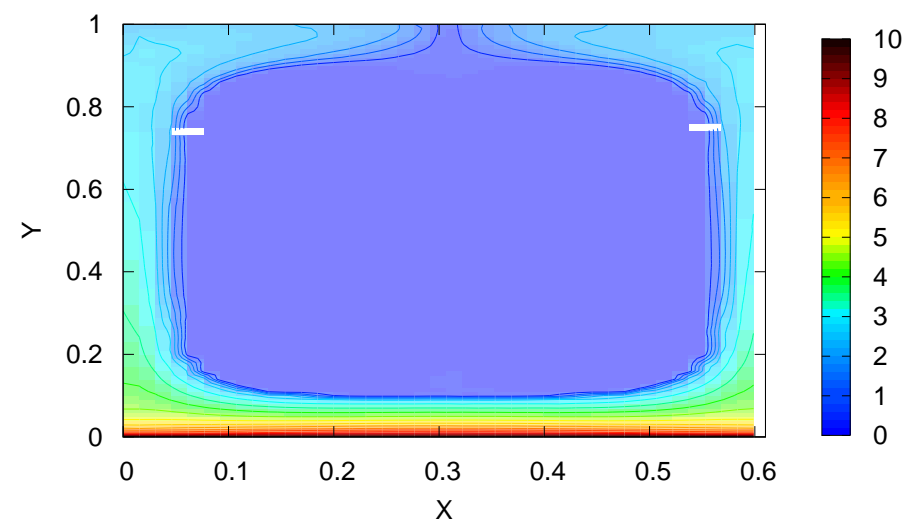

Figure 16: (color online) Contour plot of the electric charge density for $C=10, M=20$, $T=600$ and $t=20$, with the two roll structure.

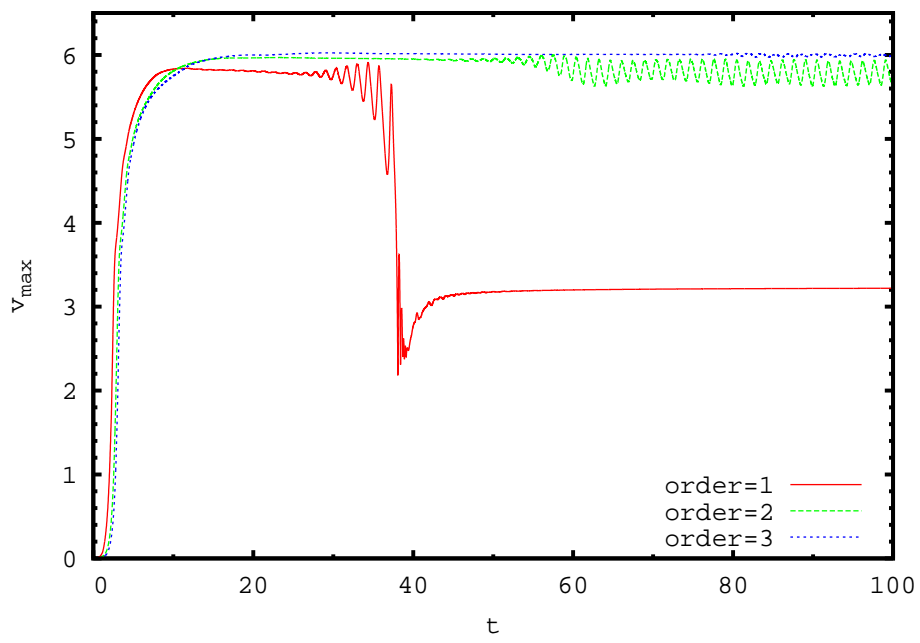

Figure 17: (color online) Evolution in time of the maximum velocity for $C=10, M=10$ and $T=300$ for three different order of polynomial approximation in the DG element. The roll becomes unstable when the order is 1 . The behaviour is different for orders 2 and 3. The oscillations start before in the former case. 